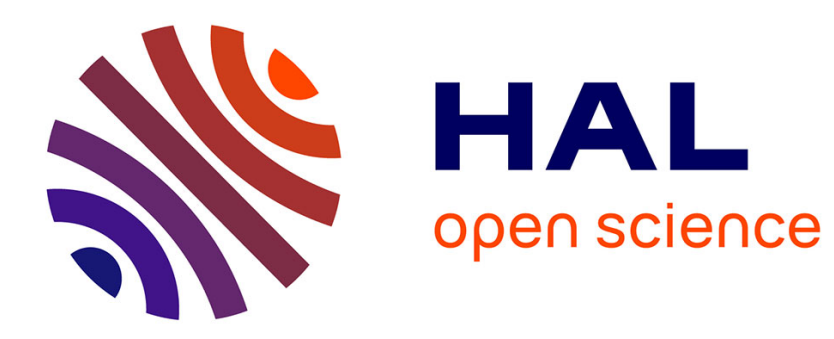

\title{
Sampling over spiraling curves
}

Philippe Jaming, Felipe Negreira, José Luis Romero

\section{To cite this version:}

Philippe Jaming, Felipe Negreira, José Luis Romero. Sampling over spiraling curves. SAMPTA 2019, Jul 2019, Bordeaux, France. hal-02436173

\section{HAL Id: hal-02436173 \\ https://hal.science/hal-02436173}

Submitted on 12 Jan 2020

HAL is a multi-disciplinary open access archive for the deposit and dissemination of scientific research documents, whether they are published or not. The documents may come from teaching and research institutions in France or abroad, or from public or private research centers.
L'archive ouverte pluridisciplinaire HAL, est destinée au dépôt et à la diffusion de documents scientifiques de niveau recherche, publiés ou non, émanant des établissements d'enseignement et de recherche français ou étrangers, des laboratoires publics ou privés. 


\section{Sampling over spiraling curves}

\author{
Philippe JAming, Felipe NEGREIRA \\ Univ. Bordeaux, IMB, UMR 5251 \\ F-33400 Talence, France \\ CNRS, IMB, UMR 5251 \\ F-33400 Talence, France \\ philippe.jamingemath.u-bordeaux.fr \\ felipe.negreira@math.u-bordeaux.fr
}

\begin{abstract}
We present our recent work on sampling along spiral-like curves [9], and discuss the main techniques. As a first result we give a sharp density condition for sampling on spirals in terms of the separation between consecutive branches. We then further show that, below this rate, the numerical stability related to the reconstruction of compressible signals when sampled along spirals is significantly limited by the amount of undersampling.
\end{abstract}

\section{INTRODUCTION}

The problem of sampling over a planar trajectory $\Gamma$ can be stated as follows: we say that $\Gamma$ is sampling for a set $\Omega \subset \mathbb{R}^{2}$ if

$$
A\|f\|_{2}^{2} \leqslant \int_{\Gamma}|f(\xi)|^{2} \mathrm{~d} \mathcal{H}^{1}(\xi) \leqslant B\|f\|_{2}^{2}, \quad \forall f \in P W^{2}(\Omega),
$$

where $A, B>0$ are constants and $\mathcal{H}^{1}$ denotes the one dimensional Hausdorff (length) measure. Here, $P W^{2}(\Omega)$ denotes the standard Paley-Wiener space

$$
P W^{2}(\Omega)=\left\{f \in L^{2}\left(\mathbb{R}^{2}\right): \operatorname{supp}(\widehat{f}) \subset \Omega\right\} .
$$

Equivalently, one aims to reconstruct a function that is supported on $\Omega$ from samples of its Fourier transform taken along a curve. This problem is relevant, for example, in magnetic resonance imaging (MRI), where moving sensors capture the anatomy and physiology of a patient.

For pointwise sampling the key quantity is the Beurling density of a set [3], which measures the average number of samples per unit volume. When dealing with continuous trajectories, however, a more meaningful metric is the average length covered by a curve [8], [12], as a proxy for scanning times. On the other hand, the understanding of sampling trajectories in terms of their length is more subtle than the discrete case, and it is less clear what can be said in full generality [8]. Nonetheless, for some particular cases - such as parallel lines - it is possible to give a complete characterization of the sampling problem. Here we give a solution for a family curves that we call spiraling. The main two examples of such curves are the collection of concentric circles

$$
O^{\eta}:=\left\{(x, y): x^{2}+y^{2}=\eta^{2} k^{2}, k \in \mathbb{N}\right\},
$$

and the Archimedes spiral

$$
A^{\eta}:=\{(\eta \theta \cos 2 \pi \theta, \eta \theta \sin 2 \pi \theta): \theta \geqslant 0\} .
$$

José Luis ROMERO

Faculty of Mathematitcs, University of Vienna

Oskar-Morgenstern-Platz 1, 1090 Vienna, Austria

Acoustics Research Institute, Austrian Academy of Science

Wohllebengasse 12-14, 1040 Vienna, Austria

jose.luis.romero@univie.ac.at

jlromero@kfs.oeaw.ac.at

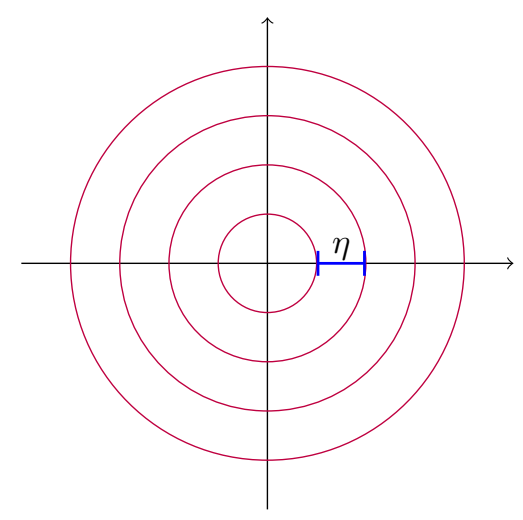

Fig. 1. Concentric circles with separation $\eta$.

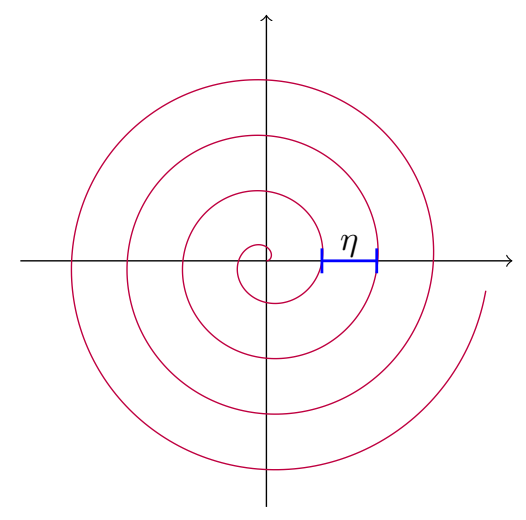

Fig. 2. Archimedes spiral with separation $\eta$.

Our first main result reads as follows.

Theorem I.1 ([9]). Let $\Omega \subset \mathbb{R}^{2}$ be a convex centered symmetric body.

(i) If $\operatorname{diam}(\Omega) \eta<1$, then the Archimedes spiral $A^{\eta}$ and the collection of concentric circles $O^{\eta}$ are sampling trajectories for $P W^{2}(\Omega)$.

(ii) If $\operatorname{diam}(\Omega) \eta>1$, then neither the Archimedes spiral $A^{\eta}$ nor the collection of concentric circles $O^{\eta}$ are sampling trajectories for $P W^{2}(\Omega)$.

In the reference case of the unit square $\Omega=[-1 / 2,1 / 2]^{2}$, 
Theorem I.1 tells us that the critical value for reconstruction over $A^{\eta}$ or $O^{\eta}$ - i.e. their Nyquist rate - is $\eta=\sqrt{2} / 2$.

We then consider slightly less dense spirals, and restrict the reconstruction problem to functions that are compactly represented in certain dictionaries. In many modern sampling schemes, such undersampling is expected to be possible because many signals of interest are highly compressible [7]. In this direction, we obtain a result for functions obeying a variation bound:

$$
\mathcal{F}(W):=\left\{f \in L^{2}\left([-1 / 2,1 / 2]^{2}\right): \operatorname{var}(f) \leqslant W\right\},
$$

where $\operatorname{var}(f):=\sup \left\{\int f \operatorname{div} h: h \in C_{c}^{1},\|h\|_{\infty} \leqslant 1\right\}$. Here, the resolution parameter $W>0$ essentially controls the number of active wavelet coefficients [6].

Theorem I.2 ([9]). Let $\eta=(1+\varepsilon) \sqrt{2} / 2$ with $\varepsilon \in(0,1)$, and $\Gamma=A^{\eta}$ or $\Gamma=O^{\eta}$. For $W>0$ set the stability margin

$$
A(\Gamma, W):=\inf \left\{\|\hat{f}\|_{L^{2}\left(\left.\mathcal{H}^{1}\right|_{\Gamma}\right)}: f \in \mathcal{F}(W),\|f\|_{2}=1\right\} .
$$

Then there exists $C>0$ such that

$$
A(\Gamma, W) \leqslant C(\varepsilon W)^{-1 / 2}\left(\ln ^{2}(\varepsilon W)+1\right), \quad \forall W>0 .
$$

Roughly speaking, Theorem I.2 says that when undersampling by a small factor $1-\varepsilon$ one can only recover functions up to resolution $W \approx \varepsilon^{-1}$ with a stable condition number. Indeed, if $W \gg \varepsilon^{-1}$, then implies that there exists a signal $f \in \mathcal{F}(W)$ with $\|f\|_{2}=1$ and almost vanishing measurements $\left.\hat{f}\right|_{\Gamma}$. See Section IV-B for an application to wavelet-sparse signals.

\section{SAMPLING SETS}

\section{A. Sampling with different norms}

For $1 \leqslant p \leqslant \infty$ we define the function space

$$
P W^{p}(\Omega):=\left\{f \in L^{p}\left(\mathbb{R}^{d}\right): \operatorname{supp}(\widehat{f}) \subset \Omega\right\} .
$$

Here we will only consider spectra $\Omega$ that are convex centered symmetric bodies, this is: compact, convex, symmetric with respect to the origin and with $0 \in \Omega^{\circ}$.

A set $\Lambda \subset \mathbb{R}^{d}$ it is said to be sampling for $P W^{p}(\Omega)$ if

$$
\|f\|_{p} \asymp\|f\|_{\ell^{p}(\Lambda)}, \quad f \in P W^{p}(\Omega),
$$

where $\|f\|_{\ell^{p}(\Lambda)}=\left(\sum_{\lambda \in \Lambda}|f(\lambda)|^{p}\right)^{1 / p}$, if $p<\infty$, and $\|f\|_{\ell^{\infty}(\Lambda)}=\sup _{\lambda \in \Lambda}|f(\lambda)|$.

We say that $\Lambda$ is separated if its separation,

$$
\inf _{\lambda, \lambda^{\prime} \in \Lambda, \lambda \neq \lambda^{\prime}}\left|\lambda-\lambda^{\prime}\right|
$$

is positive.

We are mainly interested in the standard spaces $P W^{2}$ (Paley-Wiener) and $P W^{\infty}$ (Bernstein). Further, as it happen to be, the problem of sampling in these spaces is almost equivalent. Indeed, a slight modification of [11, Theorem 2.1] gives:

Proposition II.1. Let $\Lambda \subset \mathbb{R}^{d}$ be a separated set, $\Omega \subset \mathbb{R}^{d} a$ convex centered symmetric body and $\varepsilon \in(0,1)$.

1) If $\Lambda$ is sampling for $P W^{2}(\Omega)$, then it is sampling for $P W^{\infty}((1-\varepsilon) \Omega)$.

2) If $\Lambda$ is sampling for $P W^{\infty}(\Omega)$, then it is sampling for $P W^{2}((1-\varepsilon) \Omega)$.

\section{B. Continuous vs discrete sampling}

When $p=2$ and more generally when $p<\infty$, a sampling set for $P W^{p}$ must be a finite union of separated sets. On the other hand, for $P W^{\infty}$ this need not be true. For example, in this case, $\Lambda$ could be the trajectory $\Gamma$ of a curve.

When dealing with $P W^{2}$, however, the relation between the discrete and continuous cases is not quite straightforward. For that, we provide a connection of both inequalities. The result mirrors that of [10] for sampling on the Bargmann-Fock space.

Theorem II.2 ([9]). Let $\Gamma \subset \mathbb{R}^{d}$ be a regular trajectory, and $\Omega \subset \mathbb{R}^{d}$ bounded with positive measure. Then $\Gamma$ is a sampling trajectory for $P W^{2}(\Omega)$ if and only if there exists a sampling set $\Lambda \subset \Gamma$ for $P W^{2}(\Omega)$.

The regularity assumptions in Theorem II.2 are rather mild; they allow for the image of any continuous curve - and hence in particular for Archimedes spirals and concentric circles.

\section{Sufficient conditions for sampling: the gap theorem}

The gap of a set $\Lambda$ is defined as

$$
\operatorname{gap}(\Lambda):=\sup _{x \in \mathbb{R}^{d}} \inf _{\lambda \in \Lambda}|x-\lambda| .
$$

The following result is due to Beurling.

Theorem II.3 ([3], [11]). Let $\Lambda \subset \mathbb{R}^{d}$ and $R>0$. If $\operatorname{gap}(\Lambda)<$ $1 /(2 R)$ then $\Lambda$ is a sampling set for $P W^{\infty}\left(\bar{B}_{R / 2}(0)\right)$.

The usefulness of the gap condition for spiraling sampling was first noted by Benedetto and $\mathrm{Wu}$ [2], in the context of pointwise sampling. For the spiral (I.2) and the concentric circles (I.1),

$$
\operatorname{gap}\left(A^{\eta}\right)=\operatorname{gap}\left(O^{\eta}\right)=\eta / 2,
$$

and hence Theorem II.3 implies that both $A^{\eta}$ and $O^{\eta}$ are sampling sets for $P W^{\infty}\left(\bar{B}_{R / 2}(0)\right)$ whenever $\eta R<1$. This leads to the proof of point $(i)$ in Theorem I.1. Indeed, by taking a separated subset $\Lambda \subset A^{\eta}$ (res. $O^{\eta}$ ) we can pass from pointwise sampling on $P W^{\infty}$ to pointwise sampling on $P W^{2}$ with Theorem II.1 and then to continuous sampling on $P W^{2}$ with Theorem II.2. Part 2 in Theorem I.1 is more challenging, because the theory of necessary density conditions for pointwise sampling does not apply to the length metric [8].

\section{Sets of uniqueness and Beurling's weak limit techniques}

A set $\Lambda$ is said to be of uniqueness for $P W^{p}(\Omega)$ if $f \in$ $P W^{p}(\Omega)$ with $\left.f\right|_{\Lambda}=0$ implies $f \equiv 0$. For the Euclidean ball $\bar{B}_{1 / 2 \tau}(0) \subset \mathbb{R}^{2}$, an example of a non-uniqueness set is the collection of equispaced parallel lines

$$
L_{\vec{v}, \eta}:=\left\{t \vec{v}+\tau k \vec{v}_{\perp}: t \in \mathbb{R}, k \in \mathbb{Z}\right\}, \quad \vec{v} \in \mathbb{S}^{1}, \tau>0,
$$

since it is included in the zeros of $\sin \left(\pi / \tau<x, \vec{v}_{\perp}>\right)$, which is a non-trivial function in $P W^{\infty}\left(\bar{B}_{1 / 2 \tau}(0)\right)$.

It is clear that all sampling sets must be of uniqueness, and although the converse is not true, it is possible to characterize a sampling set through the uniqueness of what are called its weak limits. A sequence of sets $\left\{\Lambda_{n}\right\}_{n}$ is said to converge 
weakly to $\Lambda$ if: for all $R, \varepsilon>0$ there exists $n_{R, \varepsilon}$ such that for all $n \geqslant n_{R, \varepsilon}$

$$
\begin{aligned}
& \Lambda_{n} \cap(-R, R)^{d} \subset \Lambda+B_{\varepsilon}(0), \\
& \Lambda \cap(-R, R)^{d} \subset \Lambda_{n}+B_{\varepsilon}(0) .
\end{aligned}
$$

We denote $W(\Lambda)$ as the collection of weak limits obtained by translates of $\Lambda$, i.e. $\Lambda^{\prime} \in W(\Lambda)$ if and only if there exists a sequence $\left\{x_{n}\right\}_{n} \subset \mathbb{R}^{d}$ such that $\Lambda+x_{n} \stackrel{w}{\rightarrow} \Lambda^{\prime}$.

Theorem II.4 ([3, pg. 345]). Let $\Omega \subset \mathbb{R}^{d}$ be a convex centered symmetric body and let $\Lambda \subset \mathbb{R}^{d}$. Then $\Lambda$ is a sampling set for $P W^{\infty}(\Omega)$ if and only if for all weak limits $\Lambda^{\prime} \in W(\Lambda), \Lambda^{\prime}$ is a set of uniqueness for $P W^{\infty}(\Omega)$.

\section{SPIRALING CURVES AND ITS NECESSARY CONDITIONS FOR SAMPLING}

We now give a concrete definition for spiraling curves. We say that a trajectory $\Gamma \subset \mathbb{R}^{2}$ is spiraling if the following conditions are met

1) (Escape cone). In some cone with vertex at the origin, $\Gamma$ can be parameterized in polar coordinates as

$$
\gamma(\theta)=\rho(\theta) e^{2 \pi i \theta}, \quad \theta \in \bigcup_{k \in \mathbb{N}}[k+\beta-\alpha, k+\beta+\alpha]
$$

for some $\alpha \in(0,1 / 4), \beta \in(0,1)$, and where $\rho \in \mathcal{C}^{2}$.

2) (Asymptotic radial monotonicity). There exists $k_{\rho}$ such that for any $\theta \in[\beta-\alpha, \beta+\alpha]$ the sequence $\rho(\theta+k)$ is strictly increasing for $k \geqslant k_{\rho}$.

3) (Asymptotic flatness). The curvature of $\gamma(\theta)$ goes to 0 when $\theta \rightarrow \infty$.

4) (Asymptotic equispacing). There exist $\eta, \rho_{0}>0$ such that

$$
\lim _{k} \rho(k+\beta)-\eta k=\rho_{0}
$$

5) (Asymptotic velocity). There exists a direction $\vec{d} \in \mathbb{S}^{1}$ non-collinear with $e^{2 \pi i \beta}$ such that

$$
\lim _{k \rightarrow+\infty} \frac{\gamma^{\prime}(k+\beta)}{\left|\gamma^{\prime}(k+\beta)\right|}=\vec{d}
$$

The number $\tau:=\eta \sqrt{1-\left\langle e^{2 \pi i \beta}, \vec{d}\right\rangle^{2}}$ is called the asymptotic separation of $\Gamma$. For short, we say that $\Gamma$ is a spiraling trajectory with asymptotic velocity $\vec{d}$ and asymptotic separation $\tau$. Note that $\vec{d}$ and $\tau$ are not necessarily unique; indeed for a trajectory $\Gamma$ there may be several possible escape cones and therefore different choices of asymptotic velocity and separation.

It can be shown that the Archimedes spiral (I.2) and the collection of concentric circles (I.1) are indeed spiraling curves. But there is a larger array of examples, some depicted below. Furthermore, spiraling curves are invariant under linear invertible transformations in $\mathbb{R}^{2}$, as well as under many nonlinear ones [9].

The key to prove point $(i i)$ in Theorem I.1 is to show that spiraling curves have a certain collection of parallel lines as a weak limit. More precisely:

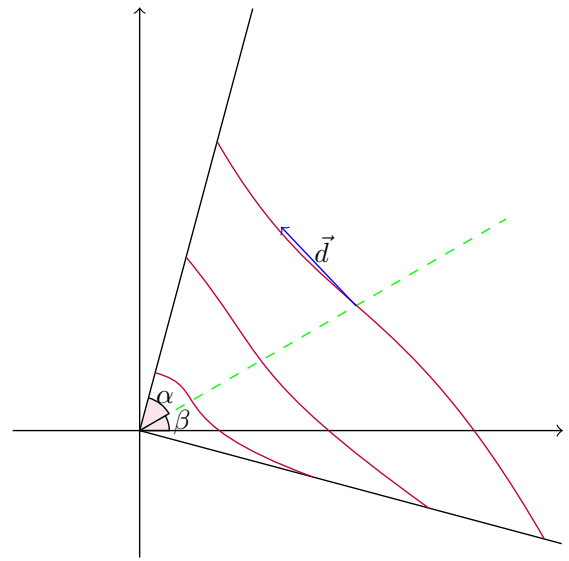

Fig. 3. Sketch of a spiraling curve inside its escape cone.

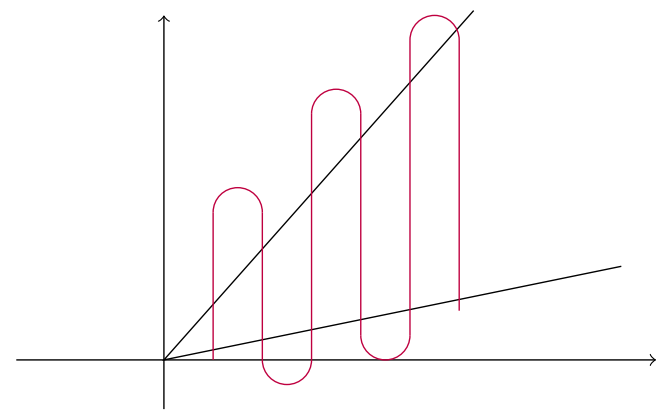

Fig. 4. A spiraling curve joining on a set of parallel segments in a plane sector.

Lemma III.1 ([9]). Let $\Gamma$ be a spiraling trajectory with asymptotic velocity $\vec{d}$ and asymptotic separation $\tau$. Then the collection of equispaced parallel lines

$$
L_{\vec{d}, \tau}=\left\{t \vec{d}+\tau k \vec{d}_{\perp}: t \in \mathbb{R}, k \in \mathbb{Z}\right\}
$$

is a weak limit of translates of $\Gamma$, i.e. $L_{\vec{d}, \tau} \in W(\Gamma)$.

Strategy of proof. The strategy to prove this lemma relies mainly on the fact that spiraling curves tend to have curvature zero, and so the branches of the curve inside the escape cone converge to straight lines. Further, the direction of such lines is

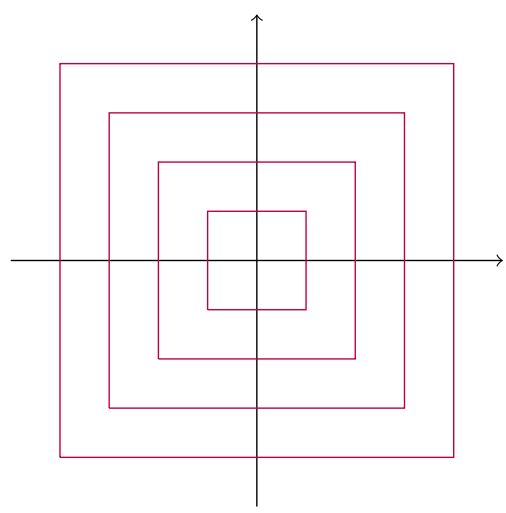

Fig. 5. A spiraling curve made of concentric squares 
given by the asymptotic velocity $\vec{d}$, and the separation comes from the asymptotic equispacing $\eta$ (or rather, after rotation, from the asymptotic separation $\tau$ ). Technically, what is behind is the Taylor expansion of degree 2 of the curve $\gamma(\theta)$.

Next, since we know how to determine the sampling spectrum of parallel lines (or rather when they are sets of uniqueness), cf. $\S$ II-D, we can then use the only if direction in Theorem II.4 to set a necessary condition for sampling on spiraling curves with the space $P W^{\infty}$. Finally, applying Proposition II.1 - with the discrete-continuous passage of Theorem II.2 - we obtain a necessary condition for sampling on $P W^{2}$. Thus point $(i i)$ of Theorem I.1 is proven.

\section{UNDERSAMPLING}

\section{A. Convergence rates for weak limits and quantitative aliasing}

Let us sketch the strategy of proof of Theorem I.2. The first step is to quantify the convergence if Lemma III.1 for the special cases of the Archimedes spiral (I.2) and the concentric circles (I.1).

As a second step, we exploit this information to construct functions with moderate variation and Fourier-controlled norm over the curve, which implies estimates on stability margins. More precisely,

Proposition IV.1. Let $\eta=(1+\varepsilon) \sqrt{2} / 2$ with $\varepsilon \in(0,1)$, and $\Gamma=A^{\eta}$ or $\Gamma=O^{\eta}$. Then given $\zeta>0$, there exists $f \in L^{2}\left([-1 / 2,1 / 2]^{2}\right)$ such that

1) $\|f\|_{2}=1, \quad\|f\|_{\infty} \leqslant C \varepsilon^{-1}$,

2) $\eta^{-1 / 2}\|\widehat{f}\|_{L^{2}\left(\mathcal{H}^{1} \mid \Gamma\right)} \leqslant \zeta$,

3) $\operatorname{var}(f) \leqslant C\left(\varepsilon^{-1} \zeta^{-2} \ln ^{4}\left(C \zeta^{-2}\right)+1\right)$,

where $C>0$ is a universal constant.

Roughly, the function $f$ in the proposition is a cut-off of the sinus function that annihilates all the parallel lines of a weak limit (as in the example of $\S$ II-D). Theorem I.2 then follows by estimating the stability margin with such $f$.

\section{B. Sampling wavelet-sparse signals}

A more explicit result in terms of sparsity can be shown from Theorem I.2 by working with the Haar-wavelet system on $L^{2}\left([-1 / 2,1 / 2]^{2}\right)$. More precisely, if $\left\{h_{j, k}\right\}_{j, k}$ is the Haar basis, we define the sparsity classes as

$$
\Sigma_{N, J}:=\left\{\sum_{(j, k) \in I} c_{j, k} h_{j, k}: c_{j, k} \in \mathbb{C}, \# I \leqslant N, 0 \leqslant j \leqslant J\right\}
$$

and the corresponding stability margin

$$
A_{N, J}(\Gamma):=\inf \left\{\|\widehat{f}\|_{L^{2}\left(\mathcal{H}^{1} \mid \Gamma\right)}:\|f\|_{2}=1, f \in \Sigma_{N, J}\right\} .
$$

We then have the following result.

Theorem IV.2. Let $\eta=(1+\varepsilon) \sqrt{2} / 2$ with $\varepsilon \in(0,1)$, and $\Gamma=A^{\eta}$ or $\Gamma=O^{\eta}$. Then for $N \geqslant 1$,

$$
A_{N, J}(\Gamma) \leqslant K N^{-1 / 6} \varepsilon^{-1} \ln ^{4}\left(K N^{1 / 3}\right),
$$

where $J=K \ln \left(\varepsilon^{-1} N\right)$, and $K>0$ is a universal constant.
Informally, this theorem says that when undersampling by a small factor $1-\varepsilon$, one can recover at most $N \approx \varepsilon^{-6}$ Haar coefficients with a stable condition number. Further Theorem IV. 2 complements related results that limit the wavelet-sparsity of discrete signals that can be sampled on unions of parallel lines [4].

The proof uses Theorem I.2 combined with the control of the approximation of bounded variation functions by Haar wavelets [6, Theorem 8.2].

\section{CONCLUSION}

We have identified the precise Nyquist rate for sampling the Fourier transform of a compactly supported function along a spiraling curve. Second, we showed that sampling slightly below this rate leads to an approximate form of aliasing that limits the possibility of effectively reconstructing compressible signals. Heuristically, successful subsampling strategies rely on avoiding regular patterns, and spirals are often mentioned as viable choices [7]. Our analysis shows, however, that perfect spirals are not adequate for significant undersampling. These results underscore the need for a certain level of randomness in sampling trajectories, and for the exploitation of finer multiscale models that apply to generic signals [1], [5].

Acknowledgments. J. L. R. gratefully acknowledges support from the Austrian Science Fund (FWF):P 29462-N35, and from the WWTF grant INSIGHT (MA16-053).

\section{REFERENCES}

[1] B. Adcock, A. C. HANSEN \& B. RomAn, The quest for optimal sampling: computationally efficient, structure-exploiting measurements for compressed sensing. In Compressed sensing and its applications, Appl. Numer. Harmon. Anal., pages 143-167. Birkhäuser/Springer, Cham, 2015.

[2] J.J. BenedetTo \& H.C. WU, Nonuniform sampling and spiral MRI reconstruction. In Wavelet Applications in Signal and Image Processing VIII, volume 4119, pages 130-142. International Society for Optics and Photonics, 2000.

[3] A. Beurling, The collected works of Arne Beurling. Vol. 2. Contemporary Mathematicians. Birkhäuser Boston, Inc., Boston, MA, 1989. Harmonic analysis, Edited by L. Carleson, P. Malliavin, J. Neuberger and J. Wermer.

[4] J. BIGOT, C. BOYER \& P. Weiss, An analysis of block sampling strategies in compressed sensing. IEEE Trans. Inform. Theory, 62(4):2125$2139,2016$.

[5] C. Boyer, J. Bigot \& P. Weiss, Compressed sensing with structured sparsity and structured acquisition. Appl. Comput. Harmon. Anal., 46(2):312-350, 2019.

[6] A. Cohen, R. DeVore, P. Petrushev \& H. Xu, Nonlinear approximation and the space BV $\left(\mathbb{R}^{2}\right)$. Amer. J. Math., 121(3):587-628, 1999.

[7] M. Lustig, D. L. Donoho, J. M. Santos \& J. M. Pauly, Compressed sensing MRI. IEEE signal processing magazine, 25(2):72-82, 2008.

[8] K. Gröchenig, J. L. Romero, J. Unnikrishnan \& M. Vetterli, On minimal trajectories for mobile sampling of bandlimited fields. Appl. Comput. Harmon. Anal., 39(3):487-510, 2015.

[9] Ph. Jaming, F. Negreira \& J.L. Romero, The Nyquist sampling rate for spiraling curves. Preprint, available as arXiv:1811.01771

[10] J. OrtegA-CerdÀ, Sampling measures. Publ. Mat., 42(2):559-566, 1998.

[11] A. OlevskiI \& A. UlanovskiI, On multi-dimensional sampling and interpolation. Anal. Math. Phys., 2(2):149-170, 2012.

[12] J. UNNIKRISHNAN \& M. Vetterl, Sampling and reconstruction of spatial fields using mobile sensors. IEEE Trans. Signal Process., 61(9):2328-2340, May 2013. 